\title{
The fourth thoracic vertebra as a radiographic landmark for the depth of right internal jugular vein catheterisation in infants: A retrospective study
}

Guoliang Liu

Beijing Children's Hospital

Jianmin Zhang

Beijing Children's Hospital

Fang Wang ( $\square$ bjetyymzk@163.com )

Beijing Children's Hospital https://orcid.org/0000-0002-4696-8733

Heqi Liu

Beijing Children's Hospital

Research article

Keywords: catheterisation, central venous catheter, infant, jugular vein, radiography, vertebra

Posted Date: October 9th, 2020

DOI: https://doi.org/10.21203/rs.3.rs-86730/v1

License: (a) (1) This work is licensed under a Creative Commons Attribution 4.0 International License.

Read Full License 


\section{Abstract}

Background $\rrbracket$ The carina is considered a reliable marker for the depth of right internal jugular vein catheterisation in infants. However, the carina is not always visible on chest radiographs, whereas vertebral bodies usually are. We performed a retrospective analysis of routine post-procedural chest radiographs of infants who had undergone congenital heart surgery and required central venous catheterisation. We evaluated the possibility of using vertebral bodies as radiographic landmarks for the identification of the depth of right internal jugular vein catheterisation.

Methods $\$ In total, 108 infants (aged 1-12 months) who underwent congenital heart surgery from the $1^{\text {st }}$ of January, 2019 , to the $30^{\text {th }}$ of June, 2019, were included. We analysed the post-operative chest radiographs of those who underwent right internal jugular vein catheterisation and assessed the visibility of the carina and vertebral bodies, as well as their positional relationship.

Results $₫$ In total, 95 children were enrolled; in 61 (64\%) cases, the carina was visible on the chest radiograph, whereas in $34(36 \%)$ cases, it was not visible. In all $95(100 \%)$ cases, the vertebral bodies were clearly visible. There was a significant difference between the rate of visibility of the carina and vertebral bodies $(P=0.01 P<0.05)$. The carina was located at the third thoracic vertebra in one case $(2 \%)$ and at the fourth thoracic vertebra in 60 cases $(98 \%)$. There was a significant difference in the position of the carina $(\mathrm{P}=0.01, P<0.05)$.

Conclusions $₫$ Most fourth thoracic vertebrae were at the same level as the carina on chest radiographs. The fourth thoracic vertebra, therefore, has potential as a radiographic landmark for the depth of right internal jugular vein catheterisation in infants when the position of the carina is not visible on the chest radiograph.

\section{Background}

Central venous catheterisation is an important cardiac surgery technique. The right internal jugular vein is the most used central venous catheter (CVC) placement site in infants; due to their relatively small size and short superior vena cava (SVC) length, determining catheterisation depth requires a high level of precision to avoid arrhythmia, heart injury, and surgical interference. ${ }^{1,2}$ When such catheterisation is too shallow, it leads to inaccurate measurement of central venous pressure and poor fluid and blood transfusion; the catheter may even be pulled out. At present, there are various methods for measuring the depth of the right internal jugular vein catheter in infants, such as transthoracic echocardiography ${ }^{3}$, intraluminal electrocardiography, transoesophageal echocardiography ${ }^{4,5}$, and surface anatomy. ${ }^{6}$ Derived formulas for calculating the depth of catheter insertion are available based on height and weight measurements. ${ }^{7}$ However, each method relies on the carina as a landmark for CVC placement in children. An anatomical measurement of infant cadavers revealed that the carina is located $22 \mathrm{~mm}$ above the junction of the superior vena cava and right atrium, ${ }^{9}$ while clinical studies have revealed that the optimal depth of CVC tip placement is at the level of the carina. ${ }^{8-10}$ The position of the carina is usually 
determined via chest radiography; however, we have observed that the carina is not always visible on chest radiographs in infants, possibly due to the condition of the patients and radiation parameters. Therefore, in some cases, it is difficult to rely on the position of the carina to determine the right internal jugular vein catheter depth in infants. However, vertebral bodies are very clear on chest radiographs; in adult anatomy, the carina is typically located at the level of the fifth thoracic vertebra. ${ }^{11}$ We are not aware of a positional relationship between infant carina and thoracic vertebra. We hypothesised that a vertebral body may serve as a radiographic landmark for the depth of right internal jugular vein catheterisation when the position of the carina is not visible on a chest radiograph.

\section{Methods}

This study was approved by the Ethics Committee of Beijing Children's Hospital, China (No. 2020-K-015) on the 20th of February, 2020. We retrospectively analysed 108 children aged between 1 and 12 months who underwent cardiac surgery at Beijing Children's Hospital, affiliated with Capital Medical University, from the 1 st of January, 2019, to the 30th of June, 2019. Informed patient consent was waived due to the retrospective nature of the study.

In this retrospective study, we aimed to analyse the relationship between the carina and thoracic vertebra in infants to determine the presence of a vertebral body, at the same level as the carina, that could be used as a radiographic landmark to guide the depth of right internal jugular vein catheterisation in infants. In total, 98 cases of right internal jugular vein catheterisation were confirmed via post-operative chest radiography. The first post-procedural chest radiograph of each child was analysed using the Carestream Picture Archive and Communication System (PACS; Carestream Health Inc., Rochester, NY, USA). The following parameters were analysed: 1) the visibility of the carina and vertebral bodies on the chest radiograph, where the position of the carina was determined as the point of division of the trachea into the left and right bronchi; 2) the positional relationship between the carina and the vertebral bodies; and 3) the position of the CVC tip on the chest radiograph.

All measurements were performed by experienced radiologists using the hospital's PACS software; all catheters were inserted under general anaesthesia. Catheterisation was performed by an anaesthesiologist, experienced in right internal jugular vein catheterisation, without measuring instruments or auxiliary devices. Due to the retrospective nature of the study, details regarding catheter placement were not clear.

\section{Statistical analysis}

Statistical analysis was performed with IBM SPSS Statistics for Mac version 21 (IBM Corp., Armonk, NY, USA). Normally distributed data are expressed as the mean \pm standard deviation, and non-normally distributed data as the median and interquartile range (IQR). Statistical significance was determined as $\mathrm{P}$ $<0.05$ using the Chi-squared test. 


\section{Results}

We reviewed the radiographs of 108 consecutive infants who underwent cardiac surgery; chest radiographs revealed that 98 of these patients underwent right internal jugular vein catheterisation. Due to the overlap of the CVC and other cardiac leads, the data of three infants could not be assessed; therefore, we enrolled and assessed 95 infants, as indicated in Fig. 1. The infants' sex, age, weight, and medical diagnosis are summarised in Table 1.

Table 1

Infant demographic characteristics

\begin{tabular}{|ll|}
\hline Variable & Value \\
\hline Age, median (IQR), months & $4(3-5)$ \\
\hline Weight, mean \pm standard deviation, kg & $5.6 \pm 1.4$ \\
\hline Sex, male/female, No. (\%) & $56 / 39(59 / 41)$ \\
Diagnosis, No. (\%) & $66(70)$ \\
Ventricular septal defect & $2(2)$ \\
Atrial septal defect & $4(4)$ \\
Tetralogy of Fallot & $4(4)$ \\
Total anomalous pulmonary venous connection & $5(5)$ \\
Patent ductus arteriosus & $5(5)$ \\
Aortic coarctation & $9(10)$ \\
Others & \\
\hline Abbreviations: IQR, interquartile range & \\
\hline
\end{tabular}

The carina was only visible on the chest radiograph of 61 cases (64\%) (not the other 34 cases (36\%)), while the vertebrae were clearly visible in all cases (100\%); there was a statistically significant difference between the rate of visibility of the carina and vertebral bodies $(P=0.01, P<0.05$; Fig. 2$)$. Analysis of the 61 cases where both the carina and vertebral bodies were visible revealed that the carina was located at the third thoracic vertebra in one case (2\%), and the fourth thoracic vertebra in 60 cases (98\%); this difference in carina location was statistically significant $(P=0.01, P<0.05$; Fig. 3$)$.

The average distance between the tip of CVC and the fourth thoracic vertebra was $11 \pm 8 \mathrm{~mm}$ in 95 children. The measurement of the vertical distance between the CVC tip and the fourth thoracic vertebra is demonstrated in Fig. 4, while the position of the catheter tip relative to the level of the fourth thoracic vertebra in the 95 patients is summarised in Table 2. In one case, a CVC tip was placed $40 \mathrm{~mm}$ below the level of the fourth thoracic vertebra. 
Table 2

Positional relationship between the CVC tip and the fourth thoracic vertebra

\begin{tabular}{|ll|}
\hline Position of the CVC tip relative to the fourth thoracic vertebra & $\%$ (No. cases) \\
\hline Above & $3 \%$ \\
\hline At the same level & $20 \%(19)$ \\
\hline Below (within $22 \mathrm{~mm}$ ) & $70 \%(66)$ \\
\hline Below (more than $22 \mathrm{~mm}$ ) & $7 \%(7)$ \\
\hline Abbreviations: CVC, central venous catheter & \\
\hline
\end{tabular}

\section{Discussion}

In this study, we retrospectively analysed the post-operative chest radiographs of infants who underwent cardiac surgery and assessed the possibility of using a vertebral body as a radiographic landmark for the identification of the depth of right internal jugular vein catheterisation. CVC insertion, including internal jugular vein catheterisation, femoral vein catheterisation, and subclavian catheterisation, is a very important perioperative technique. Currently, the most widely utilised form is right internal jugular vein catheterisation. ${ }^{12}$ The optimal timing of surgical treatment for children with certain congenital heart diseases is during infancy. Infants have a relatively large head and short neck; therefore, it is difficult to insert and place the right internal jugular vein catheter, especially in terms of the appropriate depth. There have been reports that improper CVC placement can cause serious complications in infants. ${ }^{13-15}$

According to current literature, the carina can be used as a reliable reference index for the depth of right internal jugular vein puncture. In this study, we retrospectively analysed post-operative chest radiographs of infants and observed that the carina was not clearly visible in a substantial proportion (36\%) of chest radiographs; however, the vertebral bodies were clear in $100 \%$ of cases. Moreover, we discovered that the fourth thoracic vertebra was at the same level as the carina in most cases $(98 \%)$, contrary to what is observed in adults ${ }^{11}$; this discrepancy may be due to differences in the physical development stages of infants and adults. Our results suggest that the fourth thoracic vertebra can also be used as a radiographic marker for optimal CVC positioning, which may reduce complications.

Previous studies on the depth of right internal jugular vein catheterisation in infants, e.g. that of Lopéz Álvarez et al., ${ }^{16}$ have relied on special equipment for guidance, such as those used during transoesophageal echocardiography and ultrasonography; these are expensive, difficult to operate, and not suitable for most hospitals. Other researchers, such as Uchida et al., ${ }^{17}$ have relied on chest radiography to locate the carina for the optimal placement depth of the CVC. As discussed above, this is not possible in all cases; we could only distinguish the general anatomy of the trachea, as well as that of the left and right main bronchi; only the approximate position of the carina was determined. 
The distance from the fourth thoracic vertebra to the CVC tip in our study was $11 \pm 8 \mathrm{~mm}$; in $77 \%$ of cases, the tip was below the fourth thoracic vertebra. In a previous study, it was revealed that the junction of the superior vena cava and the right atrium may be $22 \mathrm{~mm}$ below the carina in infants. ${ }^{9}$ We therefore speculated that the CVC may be too deep when placed more than $22 \mathrm{~mm}$ below the fourth thoracic vertebra, as this may cause the CVC to enter the right atrium. In this study, there were seven cases where the CVC tip was located more than $22 \mathrm{~mm}$ below the fourth thoracic vertebra; in one case, the distance from the CVC tip to fourth thoracic vertebra was $40 \mathrm{~mm}$. Theoretically, the CVC may enter the right atrium when placed at such a depth; however, such a complication was not recorded by the surgeons. In 66 cases, the catheter tip was located no more than $22 \mathrm{~mm}$ below the fourth thoracic vertebra; we speculate that the CVC tip is located in the lower segment of the superior vena cava when placed at this depth, which is reported as the most suitable depth. ${ }^{18,19}$ However, it has been reported ${ }^{20}$ that head position may have an effect on the internal jugular vein size and, thus, the catheter tip position. If placed near the fourth thoracic vertebra or carina, this effect is likely minimal; however, if the CVC is placed closer to the right atrium, a change in head position may cause the catheter to enter the right atrium, interfering with the operation and risking complications such as cardiac perforation and tamponade. Since we did not require the same head position during data collection, a closer proximity of the catheter tip to the right atrium increases the risk of complications. Based on the above analysis, the CVC should be withdrawn approximately $10 \mathrm{~mm}$, on average, to the level of the fourth thoracic vertebra; this seems to be a reliable indication of a safe CVC depth for the children analysed in this study. According to our observations, the CVC tip was located above the fourth thoracic vertebra in a safe position in three cases. However, a position that is too high would lead to inaccurate determination of the central venous pressure, increasing the risk of the catheter being pulled out.

This was a retrospective study, which resulted in certain limitations. First, post-operative chest radiography was not performed using standardised radiographic parameters for observing the position of the carina; therefore, not all carina were visible. However, regardless of the radiation parameters, vertebral bodies were clearly visible. Second, during chest radiography, the infants' head positions were not uniformly fixed, which may have affected the depth of CVC placement. Third, many studies have indicated that the insertion depth of the catheter was highly correlated with infant height $6,7,21$; however, we did not obtain infant height data from the medical records, and were therefore unable to determine whether there was such a correlation. Fourth, we did not receive feedback from the surgeon regarding the position of the catheter during the operation; therefore, we could not verify our results with more clinical information. Finally, we only focussed on infants; our results are therefore not necessarily applicable to children of all ages. These limitations highlight the need and scope for further, preferably prospective, studies on this topic.

\section{Conclusions}

In conclusion, the fourth thoracic vertebra has potential as a radiographic landmark for the depth of catheterisation in the right internal jugular vein of infants when the carina is not visible via chest 
radiography. This feature may be valuable for reducing the clinical risks associated with inappropriate CVC placement.

\section{Abbreviations}

CVC: Central venous catheter; IQR: Interquartile range; RIJV: Right internal jugular vein; SVC: Superior vena cava

\section{Declarations}

Ethics approval and consent to participate: This study was approved by the Ethics Committee of the Beijing Children's Hospital, China (No. 2020-K-015). Informed patient consent was waived due to the retrospective nature of the study.

Consent for publication: Not applicable.

Availability of data and materials: All data generated or analysed during this study are included in this published article.

Competing interests: The authors declare that they have no competing interests.

Funding: Not applicable.

Authors' contributions: FW contributed to the overall study design, data analysis, and manuscript revision. GL helped design the study, performed the experiments, collected and analysed data, and wrote the manuscript. JZ helped design the study and revised the manuscript. HL performed the experiments and collected data. All authors read and approved the final manuscript.

Acknowledgements: We thank the radiologists for their technical assistance.

\section{References}

1. Vessely TM. Central venous catheter tip position: a continuing controversy. J Vasc Interv Radiol 2003; 14: 527-34.

2. Nowlen TT, Rosenthal GL, Johnson GL, et al. Pericardial effusion and tamponade in infants with central catheters. Pediatrics 2002; 110: 137 - 42.

3. Ji-Hyun Lee., Hyo-Jin Byon., Yoon-Hyeong Choi., et Determination of the optimal depth of a left internal jugular venous catheter in infants: A prospective observational study Paediatr Anaesth 2017;27(12):1220-1226.

4. Rossetti F, Pittiruti M, Lamperti M, et al. The intracavitary ECG method for positioning the tip of central venous access devices in pediatric patients: results of an Italian multicenter study. J Vasc Access. 2015; 16:137-143. 
5. Park Y H , Lee J H, Byon H J , et al. Transthoracic echocardiographic guidance for obtaining an optimal insertion length of internal jugular venous catheters in infants[J]. Pediatric Anesthesia, 2014; 24(9):927-932.

6. Hui Xu., Xiaoqiu Zhu., Jin Li, et al . Using sternal angle as anatomic landmark for right internal jugular vein catheterisation in pediatrics .Acta Anaesthesiol Scand 2019; 00:1-5.

7. Yamamoto $T$, Schindler E . A new way to determine correct depth of central venous catheter insertion using a real-time ultrasound-guided insertion technique in pediatric patients[J]. Pediatric Anesthesia, 2019;29:368-376.

8. Yoon SZ, Shin JH, Hahn S, et al. Usefulness of the carina as a radiographic landmark for central venous catheter placement in paediatric patients. $\mathrm{Br} \mathrm{J}$ Anaesth. 2005;95:514-

9. Albrecht $\mathrm{K}$, Breitmeier D, Panning B, et al. The carina as a landmark for central venous catheter placement in small children. Eur J Pediatr. 2006;165:264-

10. Stonelake, P. A. The carina as a radiological landmark for central venous catheter tip position[J]. British Journal of Anaesthesia, 2006; 96(3):335-340.

11. Bryce Mieczkowski冈Brian F. Seavey. StatPearls [Internet]. StatPearls Publishing; Treasure Island (FL):Feb 5,2020.Anatomy, Head and Neck, Trachea.

12. Karaaslan $P, K$ Darcın, $T$ Ormecl, et al. Effects of varying entry points and trendelenburg positioning degrees in internal jugular vein area measurements of newborns.[J]. Nigerian Journal of Clinical Practice, 2018; 21(4):514-518.

13. Askegard-Giesmann JR, Caniano DA, Kenney BD. Rare but serious complications of central line Semin Pediatr Surg. 2009;18:73-83.

14. Hodzic S, Golic D, Smajic J, et al. Complications related to insertion and use of central venous catheters (CVC). Med Arch. 2014;68:300-303.

15. Nayeemuddin $M$, Pherwani AD, Asquith JR. Imaging and management of complications of central venous catheters. Clin Radiol.2013;68:529-544.

16. José M López Álvarez, Olivia Pérez Quevedo, Teresa Ramírez Lorenzo, et al. Ultrasound-guided vascular cannulation. Experience in critically-ill pediatric patients[J]. Archivos argentinos de pediatría, 2018;116(3):204-209.

17. Uchida $Y$, Sakamoto $M$, Takahashi $H$, et al. Optimal prediction of the central venous catheter insertion depth on a routine chest x-ray[J]. Nutrition, 2011; 27(5):557-560.

18. Hsu JH, Wang CK, Chu KS, et al. Comparison of radiographic landmarks and the echocardiographic SVC/RA junction in the positioning of long-term central venous catheters. Acta Anaesthesiol Scand.2006;50:731-735.

19. Z. YOON, T . J . SHIN, H . S . KIM囚et al. Depth of a central venous catheter tip: length of insertion guideline for pediatric patients[J]. 2006;50(3):355-357.

20. Gok F , Sarkilar G , Kilicaslan A , et al. Comparison of the effect of the Trendelenburg and passive leg raising positions on internal jugular vein size in critically ill patients[J]. Int $\mathrm{J}$ Clin Exp Med, 
2015;8(10):19037-19043.

21. Kim H, Jeong $\mathrm{CH}$, Byon $\mathrm{H} \mathrm{J}$, et al. Predicting the optimal depth of left-sided central venous catheters in children[J]. Anaesthesia, 2013;68(10):1033-1037.

\section{Figures}

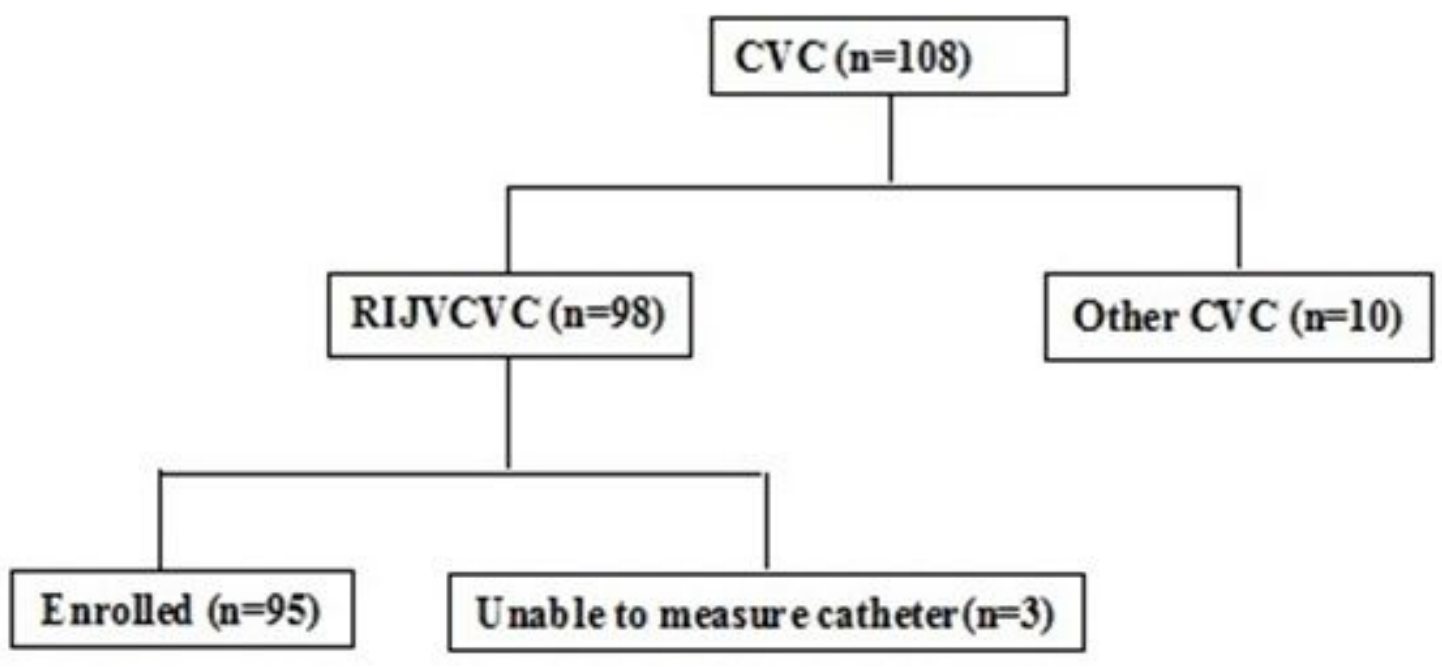

\section{Figure 1}

Details of assessed CVC cases. CVC, central venous catheter; RIJV, right internal jugular vein.

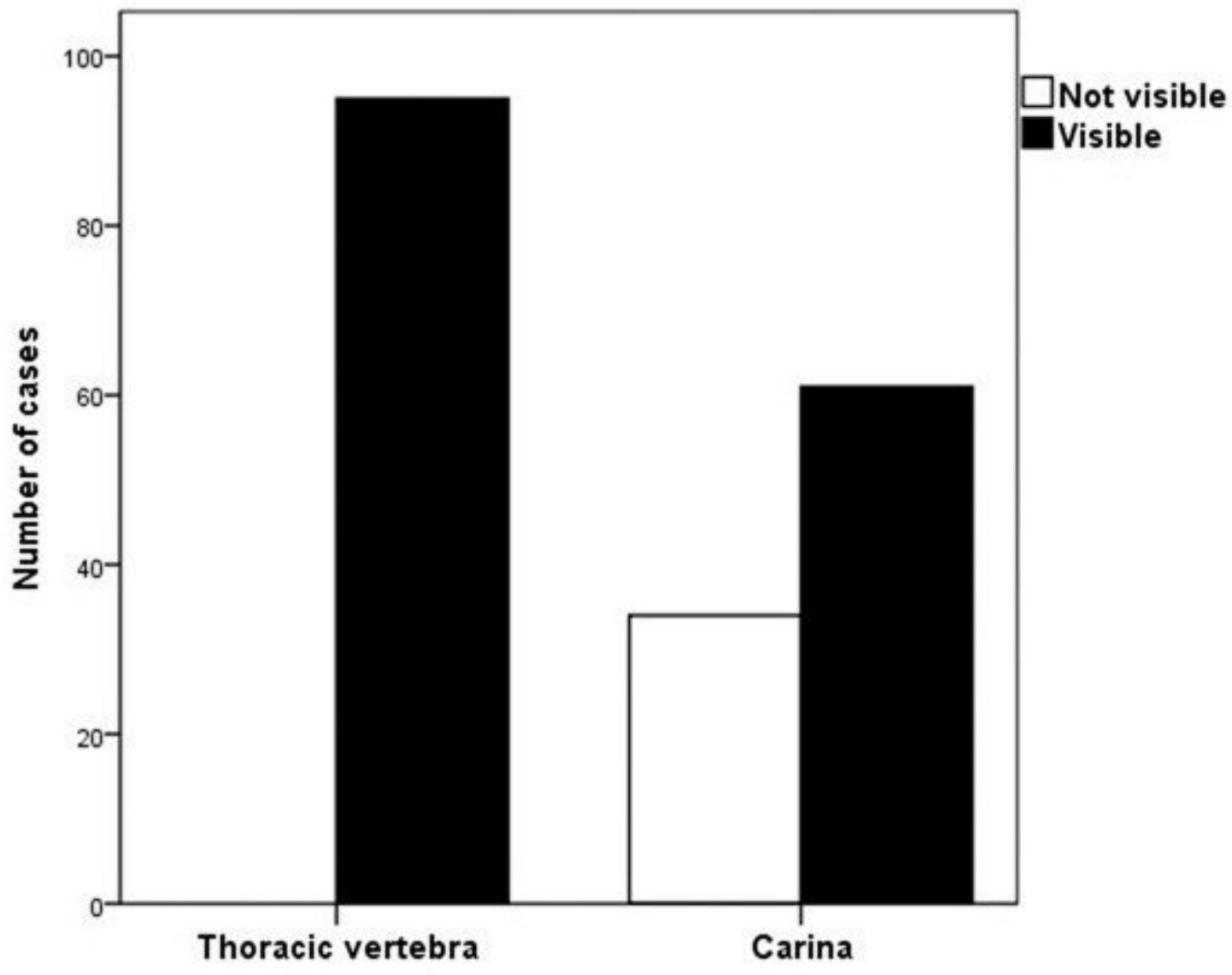


Figure 2

Comparison of the visibility of the carina and thoracic vertebra on chest radiographs. N.B.: there was a significant difference between the visibility of the carina and thoracic vertebra, $\mathrm{P}<0.05$

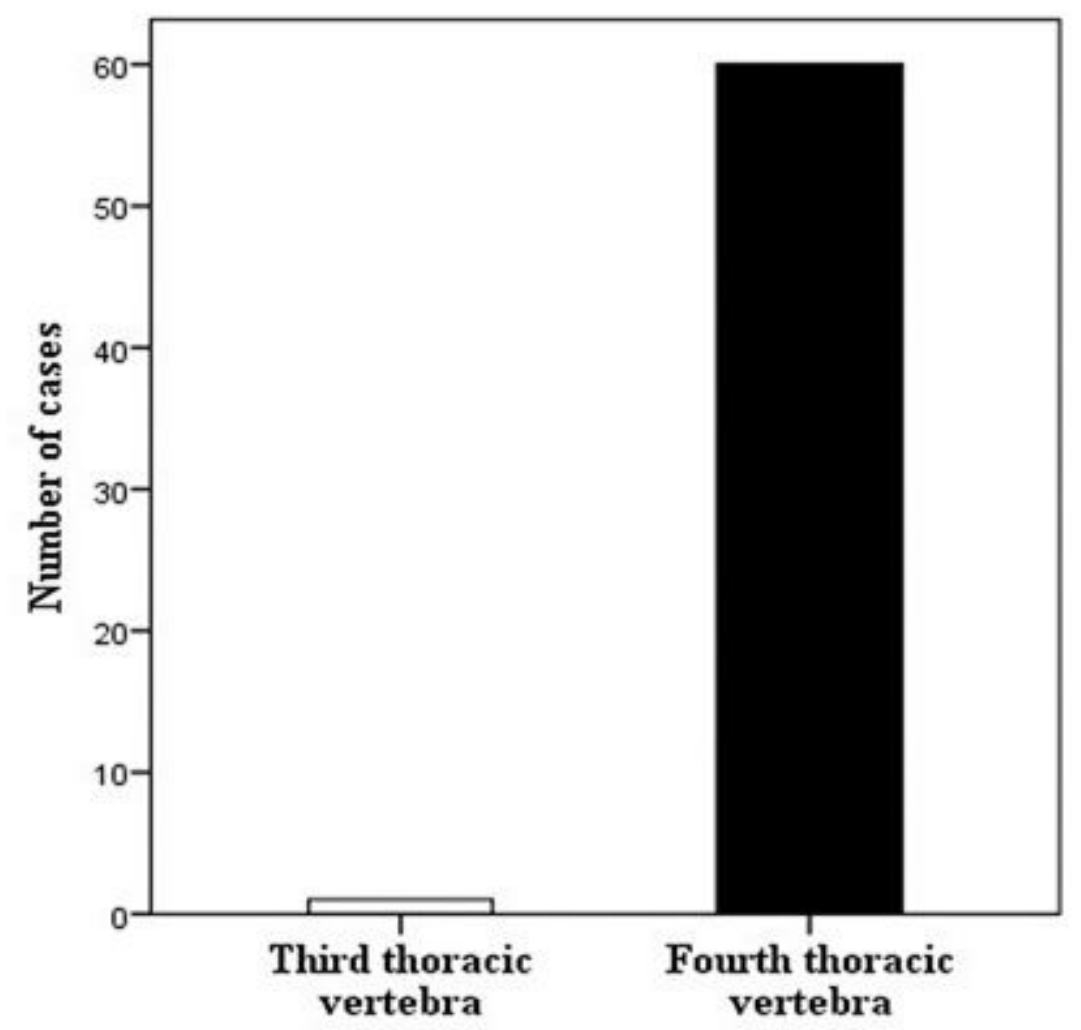

Figure 3

Positional relationship between the carina and thoracic vertebra in 61 infants. N.B.: the carina was located at the fourth thoracic vertebra more than in other locations, $\mathrm{P}<0.05$ 


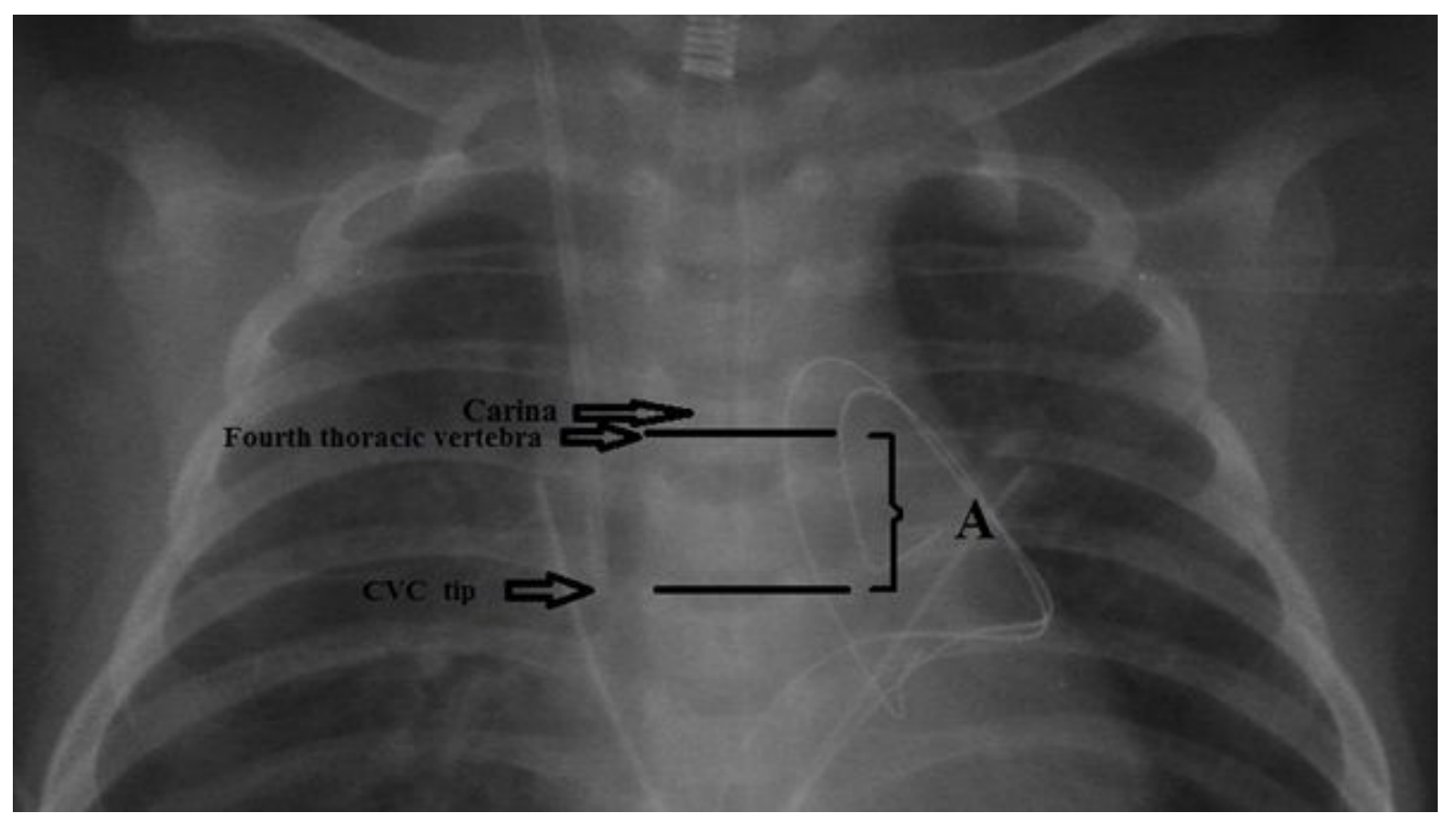

\section{Figure 4}

Measurement of vertical distance between the CVC tip and the fourth thoracic vertebra N.B.: A picture archiving and communication system was used to measure the vertical distance $(A)$ from the transverse plane of the CVC tip to the level of the fourth thoracic vertebra. CVC, central venous catheter 